\title{
Don't wait to share data on Zika
}

\author{
During public health emergencies, such as the current increase in microcephaly and neurological \\ syndromes potentially associated with the Zika virus outbreak, a rapid and coordinated response \\ necessitates the immediate sharing of data. Nature Microbiology policy is fully aligned with this imperative.
}

Much has been written and broadcast about Zika virus in recent weeks as the world wakes up to a problem that has been growing in South America for many months (for an overview, see refs 1-3). Despite this increased attention, we still know relatively little about the current outbreak and its potential impact. On 1 February 2016, following an emergency committee convened to consider the rapid spread of Zika virus and the potential links to birth defects and neurological syndromes, the WHO declared a 'public health emergency of international concern'. Raising the status of the situation will help to better coordinate necessary research into the epidemiology and virology of Zika virus and provide insight into the many questions that currently remain unanswered.

A member of the flavivirus family, Zika virus was first identified in 1947 in a monkey from the Zika Forest in Uganda (hence the name). Antibodies against Zika virus were subsequently found in human sera in 1950 and these 'footprints' were tracked as the virus spread to countries in Central and North Africa as well as Asia. Outbreaks of Zika virus in the Pacific on Yap Island and French Polynesia, in 2007 and 2013 respectively, represent the first cases outside of Africa and Asia. It was not until May of 2015 that Zika virus was detected in the Americas, with the first cases appearing in Brazil, there has been rapid dissemination ever since. At the time of writing, the virus has now been found in 23 countries in the Americas. Conditions are perfect for the spread of Zika virus, with an immunologically naive population and the presence of suitable vectors in the Aedes mosquito.

Since its initial identification, the threat from Zika virus has been considered relatively low - most infections are either asymptomatic or cause only mild symptoms, including rash, fever, joint pain, red eyes and headaches. However, this threat is now being reassessed as the latest outbreak has been linked with a surge in reported cases of microcephaly (a birth defect associated with small head size and incomplete brain development) and Guillain-Barré syndrome (progressive muscle weakness owing to damage in the peripheral nervous system). Brazil reported just 147 cases of microcephaly in 2014, but more than 4000 cases since December 2015. Assessing the potential link between Zika and the increase in microcephaly is complicated by the probable underestimation of the normal rate of microcephaly occurrence in Brazil and over-reporting during the intensive search for cases potentially linked to Zika virus. However, there is cause to take such associations seriously; the virus has been found in amniotic fluid, placenta or fetal tissue of some individuals infected. Furthermore, during the previous outbreak in French Polynesia, of around 10,000 total infections, approximately 70 were severe cases associated with neurological (Guillain-Barré) or autoimmune complications. A small number of microcephaly cases were reported following that outbreak. With the WHO estimating that there will be 3-4 million total cases of Zika infection in the next year, advice that pregnant women should avoid travelling to destinations within the current outbreak zone and take steps to reduce exposure to mosquitoes if already in an affected region, is prudent. Furthermore, scaling up efforts to control the Aedes populations is essential and will have obvious benefits, especially since these mosquitoes also carry dengue and chikungunya viruses.

An important part of a coordinated response will be for researchers and authorities in countries affected by Zika virus to publish all epidemiological and clinical data as soon as they are gathered and analysed ${ }^{4,5}$. There are some genuine barriers to the immediate publication of data that need to be overcome, including concerns over patient privacy or limited access to technology or personnel in resources-poor settings. Unfortunately, many researchers also remain fearful about making data immediately publically available for re-use by others owing to the competition to publish first and the perception that journals will consider a prior data release to impact novelty. During public health emergencies, failing to make data and analysis immediately available is to the detriment of a coordinated response, the result of which can be measured in increased morbidity and mortality.
Let us be clear here then. As has been the long-standing policy on other Nature research journals ${ }^{6,7}$, the editors of Nature Microbiology encourage the deposition in appropriate repositories and immediate release of all data, no matter the topic of the work, public-health-related or otherwise. This will not impact our editorial decision on whether or not to consider a manuscript for publication. We also encourage authors to share their initial analyses by posting a manuscript in a preprint repository, such as bioRxiv (http://biorxiv.org/). Again, this will not affect whether or not we will consider a manuscript for publication. Indeed, despite this being only our third issue, we have already published two papers ${ }^{8,9}$ for which preprints are available $e^{10,11}$. For manuscripts submitted to us that relate to an on-going public health emergency, such as Zika, if at the time of submission the data has not been made available, we will strongly encourage authors to release the data immediately rather than waiting until a paper is ready for publication. Furthermore, Nature journals have agreed to make all papers published relating to Zika virus free to access until further notice, ensuring that there are no barriers preventing the release and dissemination of data and analysis during the ongoing public health emergency ${ }^{12}$.

In the coming months through the determined effort of healthcare workers and researchers we will begin to get a clearer picture of whether Zika virus is responsible for the birth defects and neurological syndromes being reported, and if so, how. Only with immediate sharing of data can this happen on a clinically relevant time scale.

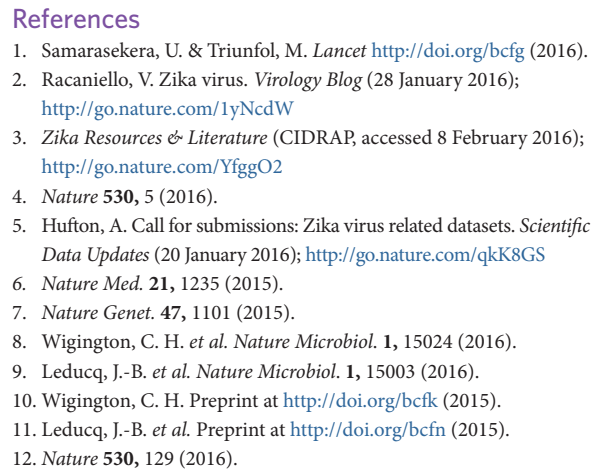

